\title{
An Improved Hybrid Active Noise Control System
}

\author{
Xing $\mathrm{Wu}^{\text {a)}}$, Jiaming Liu ${ }^{\text {b) }}$ and Binwen Fan ${ }^{\mathrm{c})}$ \\ School of Electronic and Information Engineering, Harbin Institute of Technology, Shenzhen 518000, China. \\ a) hitwx@126.com \\ b) 18601198971@163.com \\ c) Corresponding author: 943260971@qq.com
}

\begin{abstract}
In order to achieve a good noise reduction effect in a closed space, an active noise control system was built. Hybrid control systems have faster convergence and better stability than feedforward control systems and feedback control systems. We will study feedforward, feedback and hybrid control systems. This topic has been studied from the following aspects: the establishment of an active noise feedforward and feedback control model: the reference signal acquired by the reference microphone is passed through an adaptive filter to the minimum mean square error is the cost function combined with the noise residual obtained by the error microphone, which produces a secondary signal whose amplitude is close to the original noise and whose phases are opposite. It is superimposed with the original signal after being released by the secondary loudspeaker to achieve the effect of noise elimination. A hybrid control model is established to combine feedforward and feedback control systems to compare the noise reduction effects of different systems under different noise conditions. For hybrid control systems, improvements are made to adaptive algorithms or hybrid control structures to achieve better Noise reduction effect and system stability. Enclosed space noise cancellation: Based on the measurement of the sound pressure distribution of the noise in a closed space, a speaker is used to perform noise reduction on a specific area of the enclosed space.
\end{abstract}

Keywords: Active noise control, adaptive algorithms, hybrid control system.

\section{INTRODUCTION}

At present, more commonly used adaptive active noise control systems are active noise feedforward control systems and active noise feedback control systems [1]-[3]. For different application backgrounds, feedforward control systems and feedback control systems have their own advantages and disadvantages. In general, however, a feedforward control system can achieve the best noise reduction achieved by active noise control if it can provide a sufficiently efficient reference signal. However, in the case of complex noise, a good reference signal cannot be obtained and a feedback control system is needed [4]. The feedback system uses the feedback signal to estimate the input signal, so the optimum amount of noise reduction that the feedforward control system can achieve cannot always be achieved [5]. Therefore, in general, the feedforward control system is superior to the feedback control system. From the research status at home and abroad, the design of most active noise cancellation systems is based on the feedforward control system.

This research will focus on the hybrid control system, which combines feedforward control and feedback control to form a hybrid control system. The hybrid control system can combine the advantages of feedforward and feedback control systems. The use of lower-order adaptive filters can achieve better noise reduction, and can also handle more complex noise conditions, with a wider scope of application. Therefore, the research on hybrid control system has a very important significance in the field of active noise reduction. 


\section{HYBRID CONTROL SYSTEM}

\section{Analysis of the Classical Hybrid Noise Control System.}

The feedforward active noise control system uses two microphones: a reference microphone and a deviation microphone. The reference microphone is used to collect primary noise, and the deviation microphone collects the residual noise signal after the primary noise is processed by noise reduction and uses this signal to evaluate the performance of the active noise control system. The adaptive feedback active noise control system uses only one deviation sensor and can only control the predictable noise components in the primary noise. The control strategy that combines feedforward control and feedback control is called hybrid active noise control.

The hybrid active noise control system is not a simple combination of two independently designed systems. Instead, the two control structures are organically designed in one system and the design method is more flexible. The hybrid system not only maintains the advantages of the two control systems, but also complement each other to overcome the deficiencies of their respective systems. The hybrid system overcomes the problems of the stability of the feedback system and also compensates for the poor adaptability of the feedforward system to primary noise. Figure 1 is a block diagram of a classical hybrid active noise control system that uses the FxLMS adaptive algorithm [6] - [8].

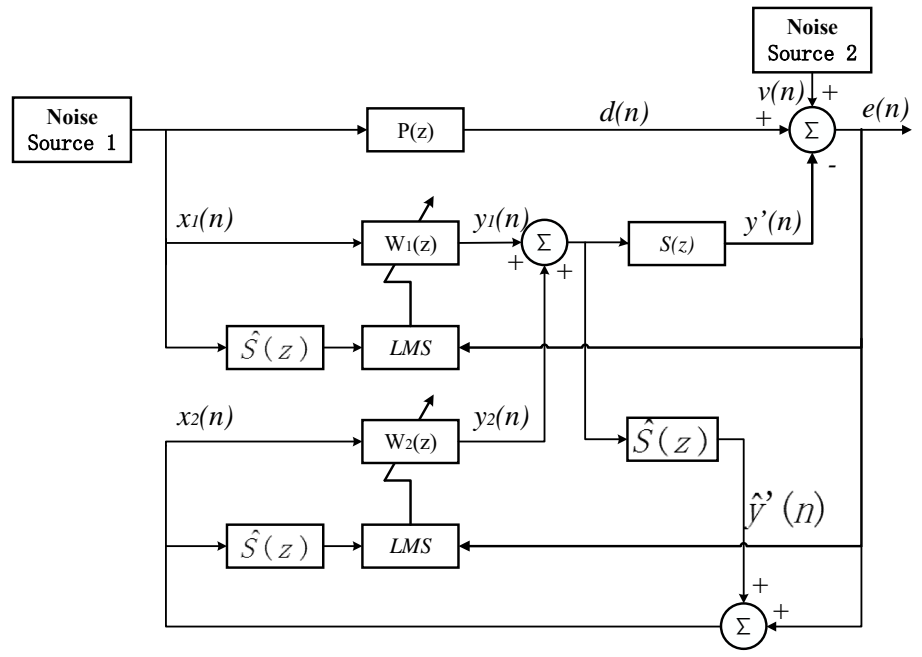

FIGURE 1. Block Diagram of A Classical Hybrid Active Noise Control System

Two noise signals $x_{1}(n)$ and $v(n)$ are uncorrelated, where $x_{1}(n)$ can be acquired by the feedforward microphone and $v(n)$ cannot be captured by the feedforward microphone, directly superimposed on the error microphone. Department. The signal finally acquired by the error microphone is a superposition of $d(n), v(n)$, and $y^{\prime}(n)$. $y^{\prime}(n)$ is a signal superimposed by the signals of $W_{1}(z)$ and $W_{2}(z)$ and passes through $S(z)$.

$$
e(n)=[d(n)+v(n)]-y^{\prime}(n)=\left[d(n)-y_{1}{ }^{\prime}(n)\right]+\left[v(n)-y_{2}{ }^{\prime}(n)\right]
$$

The error signal $e(n)$ acts simultaneously on $W_{1}(z)$ and $W_{2}(z)$. The analysis shows that $e(n)$ contains two parts, one part is needed for the feedforward part of the hybrid noise control system, that is, $W_{1}(z)$ is expected to get, but $W_{2}(z)$ is the interference signal; the other part is the opposite, It is expected that $W_{2}(z)$ is obtained, but it is an interference signal for $W_{1}(z)$.

The reference signal $x_{2}(n)$ of $W_{2}(z)$ can be expressed as:

$$
\begin{gathered}
x_{2}(n)=e(n)+\hat{y}^{\prime}(n) \\
=\left[d(n)-y_{1}{ }^{\prime}(n)\right]+\left[v(n)-y_{2}{ }^{\prime}(n)\right]+\left[\hat{y}_{1}{ }^{\prime}(n)+\hat{y}_{2}{ }^{\prime}(n)\right] \\
\approx \hat{d}(n)+\hat{v}(n)
\end{gathered}
$$


It can be seen that $x_{2}(n)$ can be divided into two parts $\hat{d}(n)$ and $\hat{v}(n)$, that is, the prediction of $d(n), v(n)$. Since the purpose of the feedback part is mainly in the hybrid active noise control system is to eliminate $v(n)$, the existence of $\hat{d}(n)$ is very bad. However, both $W_{1}(z)$ and $W_{2}(z)$ are adaptive systems and both use $e(n)$ as the error feedback signal. Although this is not appropriate, the hybrid active noise control system can still achieve convergence and achieve noise. Suppressed, but the convergence process is relatively slow.

\section{Improvement the Hybrid Noise Control System.}

Although the classical hybrid noise control system can realize the noise reduction function, it still has some problems and there is room for optimization. Figure 2 is a block diagram of an improved hybrid noise control system. There are three adaptive filters in the improved hybrid noise control system. $W_{1}(z)$ is a filter in the feedforward section for denoising $d(n)$. It relies on a feedforward microphone to obtain the noise signal $x_{1}(n) . W_{2}(z)$ is the filter of the feedback part and is used to denoise $v(n)$. The reference signal $x_{2}(n)$ is the prediction of $v(n)$, and the core of $W_{1}(z)$ and $W_{2}(z)$ is the algorithm uses the FxLMS algorithm. $H(z)$ is used to separate the residual noise $e(n)$ into two uncorrelated error signals that act on the feedforward and feedback parts, respectively.

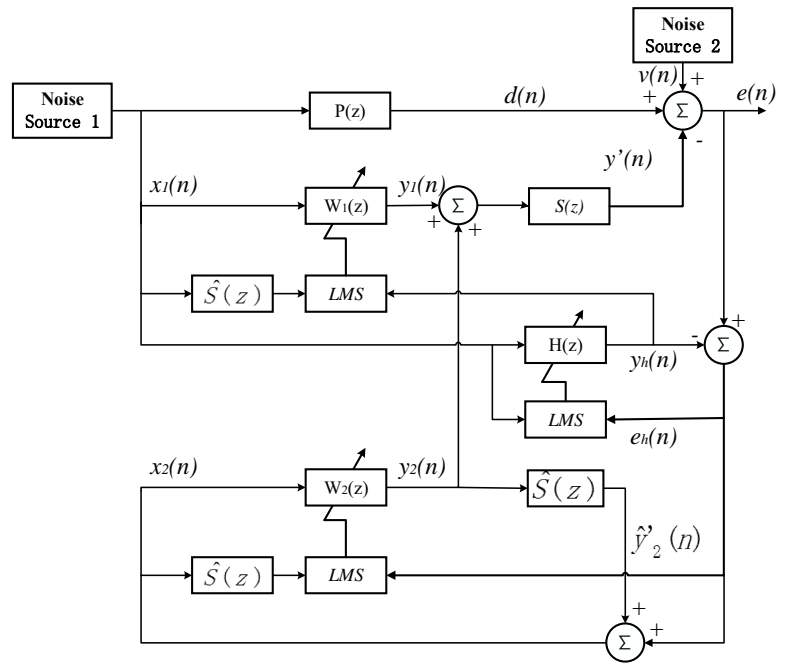

FIGURE 2. Block Diagram of Improved Hybrid Noise Control System

Similar to the classical mixed noise control system, it is not difficult to analyze the following formula:

$$
\begin{gathered}
y(n)=y_{1}(n)+y_{2}(n)=\boldsymbol{w}_{1}^{T}(n) \boldsymbol{x}_{1}(n)+\boldsymbol{w}_{2}^{T}(n) \boldsymbol{x}_{2}(n) \\
y_{h}(n)=\boldsymbol{h}^{T}(n) \boldsymbol{x}_{h}(n) \\
e_{h}(n)=e(n)-y_{h}(n) \\
x_{2}(n)=e_{h}(\mathrm{n})+\hat{s}(n) * y_{2}(n)
\end{gathered}
$$

According to the adaptive algorithm, there are three filter iterative formulas:

$$
\begin{gathered}
\boldsymbol{h}(n+1)=\boldsymbol{h}(n)+\mu_{h} e_{h}(n) \boldsymbol{x}_{h}(n) \\
\boldsymbol{w}_{1}(n+1)=\boldsymbol{w}_{1}(n)+\mu_{w_{1}} y_{h}(n)\left[\hat{s}(n) * \boldsymbol{x}_{1}(n)\right]
\end{gathered}
$$




$$
\boldsymbol{w}_{2}(n+1)=\boldsymbol{w}_{2}(n)+\mu_{w_{2}} y_{h}(n)\left[\hat{s}(n) * \boldsymbol{x}_{2}(n)\right]
$$

In the proposed method, the role of $H(z)$ is very important: it generates the required error signal for the adaptive adaptation of $W_{1}(z)$ to provide the cancellation of the correlated interfering signald $(n)$. In addition, it is used in the appropriate $W_{2}(z)$ signal is generated to eliminate irrelevant interference $v(n)$. Compared to the conventional hybrid ANC system of Figure 1, the ANC filters $W_{1}(z)$ and $W_{2}(z)$ in the proposed method use interference-free signals, and it is expected that the convergence and noise reduction performance can be improved.

Since the proposed method introduces a third adaptive filter in the existing hybrid ANC system, the computational complexity is higher than that of a conventional hybrid ANC system, and more calculations are required for each iteration. Therefore, it is necessary to study the trade-off between performance improvement and computational complexity increase.

\section{COMPUTER SIMULATIONS}

The project carried out a comparative simulation experiment of feedforward, feedback, hybrid, and improved hybrid noise control systems. Noise Source 1 consists of three single-frequency noises of $165 \mathrm{~Hz}, 290 \mathrm{~Hz}$, and $410 \mathrm{~Hz}$, and Gaussian white noise with a variance of 0.01 . These noises can be passed through. Acquired by the feedforward microphone, the Noise Source 2 consists of three single-frequency noises of $250 \mathrm{~Hz}, 350 \mathrm{~Hz}$, and $500 \mathrm{~Hz}$. These noises are directly added to the error microphone and cannot be acquired by the feedforward microphone. In the experiment, the noise sampling frequency is $3000 \mathrm{~Hz}$. The number of points is 40,000 , and the order of the secondary path modeling filter is 16 steps. When the noise is reduced, the order of each adaptive filter is 128 steps. The step length $\mu$ in the FxLMS algorithm is set as: the feedforward $\mu=0.01$, feedback $\mu=0.01$, mixing $\mu 1=0.001, \mu 2=0.001$, improved mixing $\mu 1=0.001, \mu 1=0.001, \mu h=0.01$.

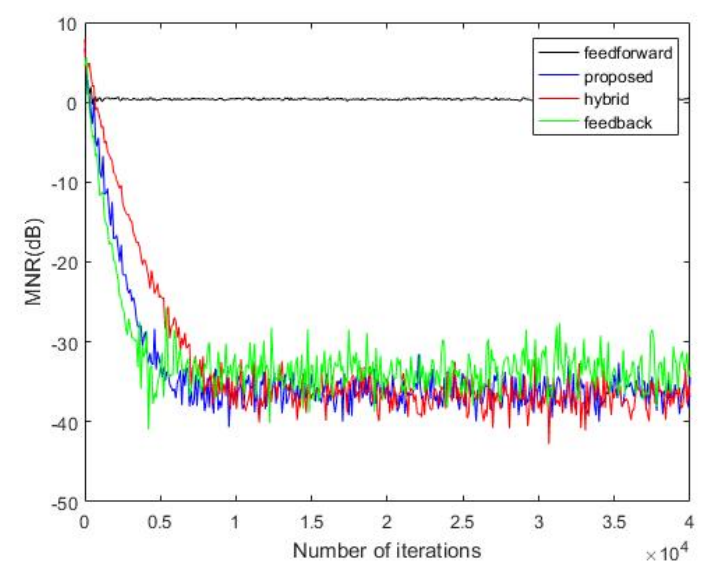

FIGURE 3. Curves for Mean noise reduction (MNR) of vatious systems

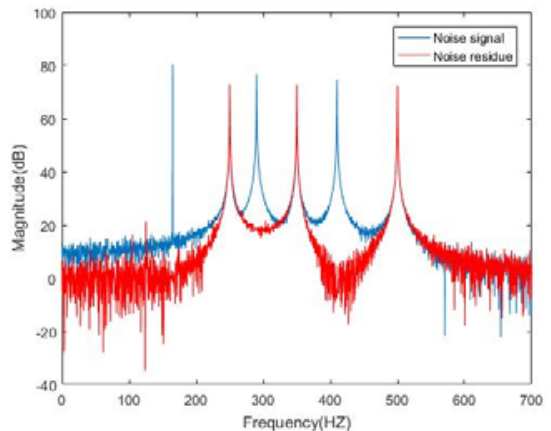

(a)

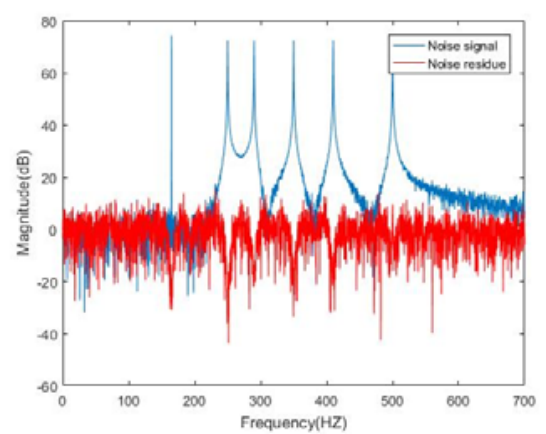

(b) 


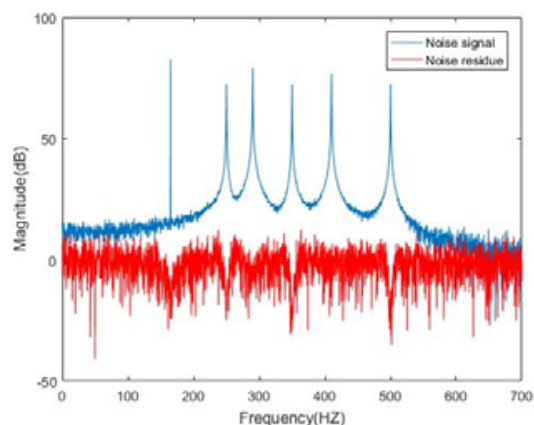

(c)

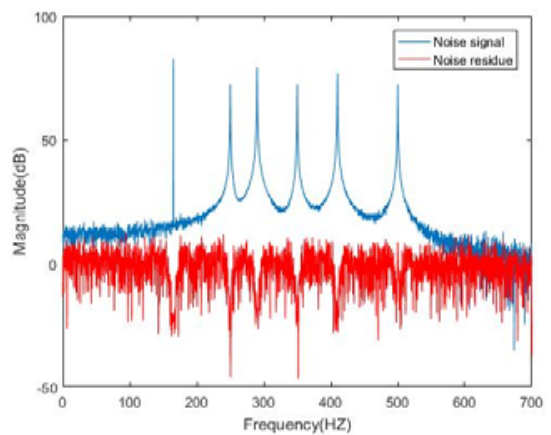

(d)

FIGURE 4. Noise Spectrum Analysis

The average noise reduction amplitude of the residual noise is shown in Figure 3. It can be observed that the noise reduction effect of the feedforward system is very poor, and the noise amplitude still maintains a large value. Figure 4(a) shows the residual noise. Spectrum analysis diagram. Since the half-frequency noise is not detected by the feedforward microphone, the cancellation signal cannot include many of these frequency components. In the final residual noise, there are very high frequency components of $250 \mathrm{~Hz}, 350 \mathrm{~Hz}$, and $500 \mathrm{~Hz}$. The rest occupies less components. The low-frequency noise, feedforward system can also play a role in suppression, for higher frequency noise, noise reduction effect becomes worse.

The residual noise spectrum analysis diagram of the feedback system is shown in Figure. 4(b). The feedback system plays a good role in suppressing the six single-frequency noises, and the convergence speed is also faster, but the remaining noise reduction effect is lower for the components. Poor, even at some frequencies, enhanced effects, exposing the limitations of their noise reduction adaptation range. After being skilled, the noise amplitude still shows large fluctuations, the performance is unstable, and the noise reduction effect is also slightly worse with the hybrid system. During the debugging process, the feedback system is more prone to divergence than other systems. For the adaptive filter's order and step size, the usable range is small, and there will be more limitations in practical application scenarios.

The residual noise spectrum analysis of the classical hybrid system is shown in Figure 4(c). It can be observed that for the six single-frequency noise and the remaining noise in a wide frequency range, the hybrid system achieves a good noise reduction effect. The amplitude of the noise is also lower, and it is more stable after convergence. The hybrid system is more stable than the feedback system. It has less dependence on the feedforward signal than the feedforward system, and it also has strong adaptability to the broadband narrowband noise. The hybrid system combines the advantages of the feed forward system and the feedback system, overcomes its shortcomings, and achieves a good noise reduction effect. However, there is still room for improvement in terms of convergence speed and stability.

The residual noise spectrum analysis of the improved hybrid system is shown in Figure. 4(d). It can be observed that the noise reduction effect of the hybrid system is not much different from that of the classical hybrid system. Both can suppress the wideband narrowband noise, and it can suppress the narrowband noise. The suppression effect is slightly strengthened, but it can be seen from Fig. 1 that the convergence speed is faster than the classical mixed noise control system. Due to its structural improvement, the improved hybrid system also has better stability.

\section{SUMMARY}

In this paper, we propose a hybrid ANC system that can simultaneously control the correlated and uncorrelated noises that appear on the error microphone. Simulation results show that this method improves the noise reduction speed and convergence speed. Due to the introduction of a third adaptive filter in a conventional hybrid ANC system, the cost to improve performance is only a small increase in computational complexity.

\section{REFERENCES}

1. Kuo SM, Morgan DR. Active noise control: a tutorial review. Proc IEEE. Vol. 87 (1999) No. 6, p. $943-73$. 
2. Sun X, Kuo SM. Active narrowband noise control systems using cascading adaptive filters. IEEE Trans Audio, Speech, Lang Process. Vol. 15 (2007) No. 2, p. 586-592.

3. Akhtar MT, Mitsuhashi W. Improving performance of hybrid active noise control systems for uncorrelated narrowband disturbances. IEEE Trans Audio, Speech, Lang Process. Vol. 19 (2011) No.2, p. 2058-2066.

4. Mokhtarpour L, Hassanpour H. A self-tuning hybrid active noise control system. J Frank Inst Vol. 349 (2012) No. 5, p. 1904-1914.

5. Wu L, Qiu X, Burnett IS, Guo Y. Decoupling feedforward and feedback structures in hybrid active noise control systems for uncorrelated narrowband disturbances. J Sound Vib. Vol. 350 (2015), p. 1-10.

6. Paleologu C, Ciochina S, Benesty J, Grant SL. An overview on optimized NLMS algorithms for acoustic echo cancellation. EURASIP J Adv Sig Process. Vol. 2015 (2015) No. 1, p. 97.

7. Shin H-C, Sayed AH, Song W-J. Variable step-size NLMS and affine projection algorithms. IEEE Sig Process Lett. Vol.11 (2004) No. 2, p. 132-135.

8. Ciochina S, Paleologu C, Benesty J. An optimized NLMS algorithm for system identification. Sig Process. Vol. 118(2016), p. 115-121. 